\title{
Petites filles mal modelées et nouveaux modèles de fillettes dans le roman français féminin contemporain
}

\author{
Christine Détrez, Anne Simon
}

The figure of the little girl in the contemporary women's novel has come a long way: the model little girl has turned into the little girl who's poorly modeled. The recurring descriptions of these girls, true tomboys, reveal the strength of socialization and the incorporation of social norms that condition behaviour from the earliest years of life. Contemporary writing develops a new "scholarly common sense", mainly fed by psychoanalysis and by sociology, that takes form through the autobiographical aspects of the works: by overcoming barriers, the little tomboy girls succeed in making a name for themselves and shaping their own identity.

Si la pauvre Sophie de la Comtesse de Ségur connaît tant de malheurs, c'est bien parce qu'elle désobéit aux normes et aux lois, rappelées à coups de punitions par sa mère, et incarnées en exemples vivants par ses cousines, les petites filles modèles. Que l'on ressente de la sympathie ou de la pitié pour Sophie, que la Comtesse ait éprouvé tendresse ou sadisme à nous raconter les bêtises et châtiments de la fillette, Sophie est, en tous les cas, fautive.

Or, en un siècle, le personnage de la petite fille « mal modelée » semble avoir envahi les romans féminins contemporains, et s'être affranchi de jugement moral. Garçon manqué, et le terme est à lui-même l'expression de toute la différence de valeur accordée aux deux sexes, ces petites filles sont des fillettes « ratées » selon les normes et les lois sociales. De la même façon que les Petit Chaperon rouge aguicheurs et indépendants des contes actuels, où c'est le loup qui regrette amèrement d'avoir croisé le chemin de ce démon en guêpière rouge, ne prennent leur relief qu'en contraste avec l'horizon d'attente de la fin du XVIIe siècle, les fillettes des romans contemporains féminins, parce qu'elles sont " ratées ", " manquées " mettent en exergue, par un effet de contrepoint, la force de l'imagerie traditionnellement associée à la figure romanesque de la petite fille. Tout se passe en effet comme si la petite fille " mal modelée " n'avait d'existence qu'en référence implicite à la petite fille « modèle », les marges et les décalages dessinant en creux l'ombre de normes qui sont loin d'avoir disparu des représentations et de l'imaginaire collectifs.

Le but premier de cet article ${ }^{i}$ est ainsi d'étudier la figure, récurrente dans les œuvres littéraires depuis les années 1990, de la mauvaise petite fille, qui dénonce les normes sociales implicites en s'en démarquant, et comment ce thème s'incarne et s'incorpore dans les descriptions physiques. Mais si cette « petite fille ratée $»$ - tant physiquement que psychiquement — innove par rapport à une 
tradition établie, il faudra également diagnostiquer dans quelle mesure de nouveaux topö̈, non dénués d'ambiguïé en ce qu'ils masquent parfois des permanences comportementales et fantasmatiques, viennent remplacer les anciens clichés. Comme la petite fille modèle, la petite fille mal modelée devient, elle aussi, un modèle (littéraire et corporel) de petite fille. Pour cet article, nous avons choisi de travailler sur certains romans féminins contemporains, sans prétendre évidemment à une exhaustivité illusoire: notre corpus est ainsi composé de Garçon manqué (Bouraoui 2000), La Tête en bas (Châtelet 2002), les œuvres d'Annie Ernaux, Dans ces bras-là (Laurens 2000), En toute innocence (Cusset 1995), La Sorcière (Ndiaye 1996), Métamorphoses de la reine (Fleutiaux 1984), Nous (Nobécourt 2002), Métaphysique des tubes (Nothomb 2002).

\section{De la petite fille modèle à la petite fille mal modelée}

Par leurs défaillances, leurs erreurs, mais aussi leurs révoltes, les petites filles romanesques contemporaines parviennent à faire éclater la naturalisation des rapports de genre et de socialisation (entendons par là la transformation en donnée de nature de ce qui relève en réalité d'une élaboration culturelle inconsciente collective et millénaire $\left.{ }^{\mathrm{ii}}\right)$. La petite fille mal modelée, par sa mise à l'index, met en effet en relief la force du modelage qui s'applique habituellement sur le corps enfantin, le dressant, le pliant, le ployant pour en faire précisément moins un corps de petite fille en tant que tel qu'un corps de femme en germe: la petite fille est une adulte en gestation, un embryon qu'il s'agit de mener à son terme social (la capacité à procréer et à entretenir le foyer, bref, à savoir quelle place tenir, et à quelle place il faut rester). Pour paraphraser Simone de Beauvoir, « on ne naît pas petite fille, on le devient $»$, cette incorporation globale étant mise au service de jeux et d'enjeux symboliques: le décalage sexué est ainsi signe d'une démarcation plus générale, témoignant par là même que le corps ne relève pas du registre de l'avoir, comme le voudrait le dualisme classique, mais du registre de l'être. Être une vraie petite fille, c'est, pour Nina Bouraoui, être vraiment française. C'est pour Annie Ernaux, être vraiment de ce milieu social plus favorisé que le sien que lui ouvre sa réussite scolaire, c'est, pour Denise/Pauliii, l'hérö̈ne hermaphrodite de Noëlle Châtelet, être vraiment une femme.

Le corps des petites filles est ainsi dressé de façon extrêmement précise par des activités sexuellement distinguées et distinguantes: dans Garçon Manqué, les garçons, accompagnés de la petite Nina en révolte, ont ainsi le droit de se rendre à la plage, de nager, de plonger ou de se mesurer aux éléments tandis que les femmes, blessées par le vent et le soleil, sont sommées, conformément aux analyses de $\mathrm{P}$. Bourdieu dans La Domination masculine, de rester à l'intérieur. Nina est ainsi « la seule fille qui joue au football » (Bouraoui 15), et son père, qui apparaît ici, à l'instar du père de Denise/Paul, comme une figure de résistance aux injonctions traditionnelles, lui apprend « le foot, le volley, le crawl », « à plonger des rochers bruns et luisants. Comme les voyous » (23). Denise Lesur, 1'héroïne autobiographique d'Annie Ernaux, délaisse ses poupées pour le vélo et le ballon (1981). Dans La Tête en bas, l'enfance du personnage hermaphrodite est elle-même scindée entre les leçons de piano supervisées par sa mère, et les 
activités sportives (trapèze, courses) avec le père, en une oscillation qui souligne dans le déroulement même de la journée, une identité clivée entre une part féminine et une part masculine dramatiquement impossibles à conjoindre.

Ces sports qui musclent trop les corps de ces fillettes montrent comment l'identité sexuée s'inscrit au ccur de la morphologie, venant redoubler les caractères sexuels primaires,

comme si ce que les physiologistes appellent les " caractères sexuels secondaires » étaient devenus les signes des rôles assignés à l'un et l'autre sexe: les « rondeurs féminines » correspondent à la fonction maternelle avec les représentations de douceur et de sensibilité et la carrure masculine est à mettre en rapport avec l'engagement dans la vie sociale avec les représentations sousjacentes d'action, de courage, de puissance. (Pagès 227)

Dans la mesure où les héroïnes ambiguës de ces romans contemporains ne parviennent pas à égaler les « vraies » filles - et l'expression est récurrente dans les romans cités, sans compter les nombreuses variations, " les toujours mignonnes ", " les gamines Ségur " (Ernaux 1981, 35) --- qu'elles croisent sur les bancs de l'école, elles trahissent la construction sociale du genre, ce qu'E. Goffman nomme la ritualisation de la féminité (1977). Les cheveux courts, les cuisses musclées de Denise/Paul, le « ventre dur ", la " poitrine musclée », les « épaules fortes » de la petite Nina sont aussitôt interprétés comme des signes d'anormalité par des parents à l'affût des moindres indices génériques, qu'ils soient biologiques (les règles, la naissance de la poitrine, la sonorité de la voix) ou comportementaux.

Ce n'est cependant pas seulement par sa morphologie que le corps se désigne comme celui d'une petite fille, mais également par ses attitudes, son « hexis »:

Il n'est pas vrai que les garçons et les filles dorment de la même manière, s'assoient ou marchent de la même manière. C'est toujours différent [...]. J'étudie, j'apprends la différence et m'applique. (Châtelet 39)

Nina se tartine de Nivéa, au grand soulagement de ses parents, qui y voient un signe rassurant de sa féminité, ignorant qu'elle mime en réalité le rituel du rasage masculin; elle apprend en outre à danser et à marcher comme un homme, « les jambes ouvertes », devant des films de Steve Mac Queen. Denise/Paul de son côté devrait avoir les cheveux longs et le dos droit des petites filles du cours de piano que sa mère observe avec regret. Elle préfère apprendre à uriner debout, comme un garçon. Par la répétition de cet acte, elle/il parvient même à changer sa morphologie, à " ne pas fuir par en dessous " (Châtelet 58). N'est-ce pas d'ailleurs ce qui menace la petite Nina? « Nina, un garçon manqué, Nina, une fille ratée. Nina, à force, il te poussera un zizi. Ou une barbichette » (Bouraoui 107). 
Le comportement vestimentaire est à ce niveau crucial, l'habit étant censé « faire 》 la petite fille, ou inversement, la « défaire ». La métamorphose évoquée cidessus de la petite Denise/Paul suit l'achat d'un costume de garçon, et est attribuée « aux vertus du pantalon gris de laine vierge ». La petite Nina, de la même façon, refuse de quitter le pantalon qui lui a été prêté par un ami, comme si celui-ci, par une sorte d'osmose ou de métonymie lui conférait, enfin, la virilité. Ici encore s'opposent les vraies fillettes, à rubans et falbalas, et ces petites filles ratées qui, comme Annie Ernaux dans La Femme gelée ${ }^{\text {iv }}$, refusent les jupes et les robes, de même que l'ensemble des attributs censés être naturellement féminins, tout le " tralala » comme le nomme Denise/Paul, vécu comme un véritable déguisement: «Porter de vraies chaussures, des chaussures qui ferment. Ne pas manger avec ses doigts. Dire bonjour et merci. Porter des robes. Se taire " (Bouraoui 95). La Denise de La Tête en bas, de la même façon, a la sensation de se déguiser, de s'accoutrer, bref de se travestir en fille, et doit apprendre à s'asseoir, à marcher, à courir avec une jupe (cette jupe dont Pierre Bourdieu dans La Domination masculine, à la suite de nombreux travaux d'historiens de la mode et des comportements, a mis en relief le caractère contraignant). D'instrument de séduction, la robe devient instrument de torture et de déformation de l'identité profonde: Denise/Paul la sent « battre sur ses jambes comme des gifles, coller à la peau comme des insultes » (Châtelet 48). Si les deux fillettes de La Sorcière acceptent en apparence la transmission des capacités génésiques que leur inculque leur mère (symbolisée par la faculté de verser des larmes de sang, qui évoquent une association entre les menstrues et la douleur), elles résistent de même à cette transmission par une androgynie vestimentaire et comportementale.

L'espace lui-même s'organise, au gré d'une géographique symbolique, en territoires masculins ou féminins. Pierre Bourdieu l'avait montré pour la maison kabyle: dans un jeu de correspondances entre monde des humains, monde des objets et langage, se réfractent les valeurs et les représentations associées à chaque genre. L'espace est un pense-bête, une « sorte de livre où toute chose parle métaphoriquement de toutes les autres et dans lequel les enfants apprennent à lire le monde, [qui] se lit avec tout le corps » (Bourdieu 1980, 129). Le monde est ainsi perpétuellement traversé de frontières qui, invisibles, en sont d'autant plus efficaces. Elles régissent à la fois l'univers domestique, jardin contre cuisine, et le dehors du foyer, intérieur contre extérieur. De la fenêtre de la cuisine ainsi, espace par excellence dévolu au féminin, la mère de Denise/Paul regarde sa fille jouer avec son père dans le jardin, tandis que la sorcière de Marie Ndiaye contemple mélancoliquement la course androgyne des deux fillettes, vêtues de shorts et de tee-shirts, qui s'en vont rejoindre le groupe des petits voisins. Chez Nina Bouraoui, plusieurs occurrences font de la vitre de la voiture un obstacle infranchissable, qui finit, comme c'était déjà le cas pour sa mère au début du roman, par séparer Nina des petits garçons jouant dans une rue devenue interdite. Ces clivages spatiaux prennent tout leur sens normatif au sein de l'univers scolaire, où, mieux qu'ailleurs sans doute puisqu'il s'agit là d'une institution au sens le plus fort du terme ${ }^{v}$, on apprend à être qui l'on doit être, et à se tenir, pour reprendre un terme cher à Ernaux, à sa « place »: c'est à l'école et à la confession 
que Denise Lesur apprend qu'elle est différente des autres, de ces vraies petites filles qui n'ont pas de pensées perverses et dont les mères font les lits tous les matins (Ernaux 1974, 59). À l'école, les travées séparent les filles des garçons (et les Français des Arabes dans le cas de Nina Bouraoui), la cour comporte l'espace de la corde et celui du ballon, les toilettes enfin sont évidemment différenciées. C'est dès lors en franchissant la porte des toilettes des garçons que Denise/Paul ose la première fois, se prétendre garçon. Que ce soit par ses protestations ou par ses humiliations, la petite fille dénonce l'intériorisation des modèles de comportements sexués, dans ces instances de socialisation que sont famille et école, qui, en temps « normal », parce que ces lois sont silencieuses « invisibles dans l'odeur douce de nourriture et de cire flottant dans les escaliers, dans la rumeur des récréations, le silence traversé par les gammes d'une leçon de piano particulière " (Ernaux 1997, 84), s'imposent « avec l'évidence du naturel et le naturel de l'évidence, c'est-à-dire délestée de ses codes arbitraires »(Blöss 2). La plupart des oeuvres d'Annie Ernaux, et notamment La Honte, sont ainsi fondées sur la mise au jour des codes familiaux et scolaires, ces « tables de la loi » (73): de leur décalage naît la honte, signe de la violence silencieuse avec laquelle ils s'appliquent. Ainsi, la récurrence du verbe « apprendre " chez ses fillettes pour qui, justement, être une fille n'est pas évident, met en relief le processus, souvent implicite, de socialisation. À la violence des rapports de force exprimés par un titre comme Moi, Christiane F, 13 ans, droguée, prostituée répond alors la violence symbolique de la norme sociale dans la variation effectuée par Noëlle Châtelet: « moi, Denise, 12 ans, fille, mais aussi garçon » (57).

Enfin, l'identité de genre s'avère indiscutablement sociale dans la mesure où elle passe par les mots, par la langue. Le langage est étroitement associé à l'identité et à la corporéité: Nina tente désespérément de maitriser l'arabe, l'héroïne de Camille Laurens est fascinée par la langue du père, la langue des hommes, qui ne s'embarrasse pas de métaphores et de poésie, " une langue verte, une langue qui en a » (127). Quant à Denise Lesur, elle apprend à l'école une nouvelle langue, le «bon » français, dépouillé du patois et des expressions familières et familiales, en même temps qu'un nouveau comportement corporel. Les historiens et sociologues l'ont montré, comme on dresse les corps, on châtie le langage et on en polit les aspérités. Corporéité et langue sont indissociables: être une petite fille, c'est aussi parler comme une petite fille, être « polie » et surtout, se taire.

Les mots sont d'ailleurs souvent ceux des autres: l'identité de petite fille dépend de la désignation d'autrui. Ce sont les mots qui créent l'identité, et notamment le nom propre, qui fonctionne comme un véritable indexateur de genre: Nina s'invente un prénom masculin, Ahmed, et son père la baptise du surnom de Brio. Une nouvelle étape dans la conquête de soi comme homme, dans cette naissance " tête en bas ", est franchie lorsque l'amie de Denise la rebaptise du nom de Paul, alors qu'officiellement, pour l'état civil, elle reste une fille, et est désignée comme telle par ses papiers d'identité (par sa carte d'« identité » précisément...). Le nom propre a donc bien une fonction d'embrayeur, non plus simplement grammaticale mais sociale. 
Mais cette schizophrénie onomastique et éthologique est vécue par de nombreux tiers comme une subversion insoutenable et un danger pour la société. Les personnages de Nina et Denise/Paul, qui racontent la même méprise, celle d'être prise pour un garçon dans la rue — et même une forme accomplie de garçon, dont on souligne la beauté: « tu es beau » (Bouraoui 36), « oh, le beau petit garçon " (Châtelet 18) - ne tardent pas à la vivre comme une réprobation dès lors que l'erreur d'optique est rétablie. Si elle peut susciter d'abord le rire chez la mère de Denise/Paul, la méprise devient vite un signal d'alarme: « regarde ta fille, Maryvonne. Regarde donc. Ouvre les yeux. Son allure dans la rue. Les réflexions des gens. Du garçon de café. De la vendeuse » (Bouraoui 64). Le trouble semé dans le corps social est tel que naissent ainsi des craintes fantasmatiques, comme celle que la fréquentation de cette étrange petite fille rende les petits garçons... homosexuels.

L'écart par rapport à la norme, devant lesquels se sentent impuissants les parents, les conduit ainsi à avoir recours à la médecine, autre lieu du panoptisme social et du pouvoir sur les corps, puisque son regard s'avère capable de traverser les chairs pour les transformer en agissant cette fois non plus sur les comportements mais sur l'organisme lui-même, de restaurer l'ordre et d'assurer, dans la violence la plus extrême puisque la plus intérieure, la diffusion de la norme. Denise/Paul est ainsi traitée à coups de piqûres hormonales visant à créer morphologiquement cette femme qu'elle récuse en elle (et effectivement vont lui pousser des seins), Nina, à chaque été en France, passe un examen médical, " pour vérifier [sa] vie algérienne », Marie dans En toute innocence de Catherine Cusset est suivie, après son viol, « par des psychiatres des médecins » garants de sa normalité. Une fois passé l'âge de la puberté, à dix-neuf ans, l'héroïne se trouvera d'ailleurs à nouveau confrontée à une psychanalyste (qui appartient donc à la « race de profanateurs ») qui la violentera en lui parlant de son anormale virginité (comme s'il y avait un âge critique pour la défloration).

Ce sont ainsi les regards des autres qui créent les identités, et, pour reprendre un titre de Jean-Claude Kaufmann, les regards des hommes qui créent les corps de femme (1995). Regard de désir, nous y reviendrons, mais avant tout regard de l'homme par excellence, le père. À ce stade, c'est littéralement de reconnaissance qu'il s'agit: tout se passe comme si pour être vues par le père, il fallait se comporter comme un garçon, et devenir garçon. Les pères des hérö̈nes de Catherine Cusset et de Camille Laurens (Dans ces bras-là), quand on leur demande s'ils ont des enfants, répondent par la négative. Ils n'ont que des filles (c'est d'ailleurs le père qui chez les deux auteures choisit le prénom des filles, en l'occurrence chez Laurens des prénoms androgynes, Camille et Claude). Paul, celui qui fut petite fille dans La Tête en bas, attribue cette hésitation de son corps à la déception et la colère ressenties par son père à la vue de ce bébé en layette rose ( $«$ il était dans une telle fureur que ma mère s'est mise à pleurer et a regretté aussitôt et le bavoir rose, et que je sois une fille »), et dans un jeu de retournement, c'est, victoire, au père d'être reconnaissant: « quelque chose me dit que mon père m'est reconnaissant des efforts inaccoutumés que j'ai dû déployer pour devenir son Paul, son Paulo. Il en mesure le prix » (Châtelet 72). La figure 
de la petite fille telle que la dessine l'écriture féminine contemporaine est celle d'un " ratage » social, d'un écart par rapport à la norme. En ce sens se révèle la force de la socialisation et de l'incorporation dans la construction sociale du genre, qui n'est elle-même qu'une manifestation, une symbolisation du rôle social dans son aspect plus général. Rien d'étonnant ainsi à ce que ces personnages de petites filles ratées vacillent entre deux identités, et s'avèrent des hybrides ou des monstres: hermaphrodite, métisse franco-algérienne, ou transfuge de classe. Il semblerait alors que l'on assiste à un nouveau " sens commun savant ", où l'écriture littéraire absorbe les discours sociologiques autant qu'anthropologiques ou psychologiques. Certaines pages semblent ainsi tirées tout droit de classiques de sociologie. Par exemple, "A table aussi, c'est différent. Mon père boit à grandes lampées, ma mère sirote. Mon père avale, ma mère grignote, la bouche en avant "): cette citation de La Tête en bas n'est pas sans rappeler la description par Pierre Bourdieu, dans La Distinction, des façons de manger attribuées quant à lui aux classes sociales. De la même façon, les écrits d'Annie Ernaux sont nourris de sociologie.

Sur un autre plan, que l'on pense aux jeux de mots psychanalytiques faits par Camille Laurens, où le viol par l'oncle, objet d'un déplacement et d'une somatisation, se métamorphose et s'intériorise sous la forme d'un " furoncle » et d'un « ongle incarné », et à son analyse de la gestation de la petite fille comme relevant d'une transmission du même et d'une incorporation en chaîne (" une fille est toujours à l'intérieur d'une femme », 150) ${ }^{\text {vi }}$. De même, chez Noëlle Châtelet, l'explication apportée a posteriori par Denise à son hermaphrodisme, perçu comme la conséquence des pleurs de la mère et de la colère du père, relève clairement d'un fantasme analytique. Dernier exemple, Anne, l'adolescente de $\mathrm{Ce}$ qu'ils disent ou rien, partagée entre les règles de sa famille et celles de l'école, écartelée entre deux codes inconciliables dont on a montré plus haut la violence normative, somatise... par l'aménorrhée, véritable incorporation de la disparition des règles.

La jeune fille rangée, ancienne figure emblématique d'un certain féminin (que Colette, Beauvoir, Sarraute, Duras ou Yourcenar ont certes dépecé au fil de leurs récits), n'a littérairement plus lieu d'être de nos jours. De façon troublante, et par un phénomène d'inversion typologique, la petite fille mal modelée ne devient-elle pas en retour la manifestation de représentations sociales, et n'est-elle pas condamnée à devenir un modèle?

\section{La fillette mal modelée, un nouveau modèle de petite fille?}

Nous l'avons vu, la figure du père est centrale dans la socialisation de ces petites filles modernes: toutes veulent attirer le regard paternel, et se démènent de tous leurs muscles pour y parvenir. Dans cette variation du complexe d'Électre, ce n'est pas par leur féminité que ces enfants veulent séduire le père, mais par leur capacité à devenir comme lui, un petit garçon. Nulle concurrence avec la mère en ce cas. Bien au contraire, le modèle maternel est rejeté, voire méprisé, avec plus ou moins de violence selon les œuvres: les petites filles de La Sorcière, de Marie Ndiaye, témoignent d'un dépassement foudroyant des capacités maternelles et un 
envol final du foyervii, au sens littéral du terme puisqu'elles se sont transformées en oiseaux. Quant aux petites ogresses dans « La femme de l'ogre » de Pierrette Fleutiaux, elles appartiennent sans équivoque à l'univers, trouble et ambigu, de leur père: la scène de la dévoration, danse d'amour et de mort, est en ce sens extrêmement sensuelle - le démembrement et la mastication opérant une fusion des chairs et le ventre de l'ogre, double mortifère de la matrice féminine, retrouvant son ambivalence séculaire. Cette éviction du modèle maternel est en passe de devenir un cliché beaucoup moins nouveau qu'il n'y paraît. Il entérine en effet, sous couvert d'une libération de la petite fille par rapport à celle qui reste censée devoir assurer la transmission des potentialités génésiques, l'image de la mère coupable qui ne parviendrait pas à assumer son rôle (c'est le cas chez Noëlle Châtelet, Camille Laurens, Catherine Cusset, Lorette Nobécourt, ou Amélie Nothomb), soit qu'elle soit trop possessive, inhibante ou injuste, soit qu'elle s'avère incapable d'aimer sa fille « correctement ». Dans Nous de Nobécourt, Geneviève s'avère une mauvaise mère qui devra être libérée du poids insupportable d'une maternité non voulue par sa propre fille, qui a su quant à elle, en « bonne » mère, aimer la fillette qu'elle a mise au monde (et partant racheter une naissance ratée). De même, les deux gouvernantes japonaises qui se partagent l'éducation de l'inénarrable fillette de Métaphysique des tubes, représentent les deux faces en apparence inconciliables de la « bonne » mère au sens classique du terme (Nishio-san est la nourrice, concrètement puisqu'elle se sacrifie en laissant le « tube » lui dévorer son assiette, et symboliquement en la nourrissant, comme dirait Perrault, de berceuses, de contes et de paroles assurant la transmission de la véritable langue maternelle de l'héroïne, à savoir le japonais) et de la " mauvaise » mère, indifférente, froide, et mortifère, la seconde naissance de l'héroïne parvenant, à la fin du roman, à rassembler les trois femmes dédiées à son éducation (les deux gouvernantes et la mère biologique). La petite fille ne pourrait donc se libérer qu'au prix d'un enfermement de la mère dans son rôle traditionnel et domestique ${ }^{\text {viii }}$...

Par ailleurs, l'érotisme est au centre de bien de ces récits contemporains. Paradoxalement cependant, les petites filles dessalées qui en sont les protagonistes ne sont pas toujours libérées par un apprentissage personnel de la sexualité. Parmi le corpus choisi (Cusset, Laurens, Bouraoui, Nobécourt), nombreux sont les personnages de petites filles qui se voient l'objet d'attouchements plus ou moins poussés de la part d'adultes masculins, vécus le plus souvent comme une violence par les héroïnes (chez Cusset, la petite fille semble davantage souffrir dans sa chair et son orgueil que psychiquement; mais cette scène primitive à tous les sens du terme puisqu'elle est placée au début du roman joue un rôle fondamental dans l'impossibilité de la constitution du soi en femme qui forme le centre du récit, puisque Marie peine ensuite à perdre sa virginité...).

Plusieurs raisons sur ce lien paradoxal entre la fillette et la violence sexuelle peuvent être avancées. La première est d'ordre littéraire: le viol est une facilité narrative en ce qu'il promeut de l'« action » dans des récits intimistes où le social, peut-être parce qu'il est perçu par les auteures comme relevant d'un genre littéraire dépassé (roman picaresque à la Céline, roman d'aventure à la 
Malraux, roman social réaliste et naturaliste du dix-neuvième siècle), ne joue explicitement aucun rôle majeur, sauf chez Ernaux (sur ce paradoxe de l'action dans des romans féminins où " traditionnellement », il ne se passe rien, voir Didier Heinich). Le viol constituerait dès lors une sorte d'effet de réel ou une légitimation de l'entreprise d'écriture: le livre a une raison d'être " importante " et peut donc se nourrir de lui-même, sans avoir à en passer par une dialectisation directe des problèmes de société. Ce qui fait cependant question, de notre point de vue sociologique, est bien entendu que dans l'ensemble, il y a en France davantage de petites filles non soumises à des sévices que de petites filles violées: la petite fille " normale » n'apparaît donc pas comme un " sujet » intéressant en soi. Il lui faut un « plus » charnel dramatique pour valoir comme objet d'écriture.

La seconde raison relève d'une intériorisation de plusieurs discours issus des sciences humaines - anthropologie, psychanalyse, études féministes. Conformément à la propension - légitime, il va sans dire! — de la société occidentale actuelle à légiférer dans le sens d'une protection accrue de l'enfant, l'attouchement sexuel renvoie, dans les textes littéraires actuels, à un acte d'hybris, alors qu'il pouvait auparavant être parfois perçu comme relevant d'un apprentissage et d'un accès à la connaissance du corps (le sien propre ou celui des adultes). Il n'est évidemment pas question ici de faire l'apologie de la pédophilie, qui reprend précisément à son compte la notion plus que suspecte d'initiation, mais de mettre en exergue un certain nombre d'évolutions et de paradoxes. Évolution, au sens où, chez des auteures comme Duras ou Yourcenar, le rapport entre sexualité adulte et sexualité enfantine n'est pas aussi tranché que dans les romans contemporains. Marguerite Yourcenar ainsi s'insurge contre une certaine diabolisation de l'interaction sexuelle entre l'adulte et l'enfant: mentionnant, dans Quoi? L'éternité, les caresses de son cousin X, elle précise qu'elle sentait « vaguement qu'en lui quelque chose avait eu lieu ». Mais, poursuit-elle,

je n'avais été ni alarmée, ni froissée, encore moins brutalisée ou blessée. Si je consigne ici cet épisode si facile à taire, c'est pour m'inscrire en faux contre l'hystérie que provoque de nos jours tout contact, si léger qu'il soit, entre un adulte et un enfant pas encore ou à peine pubère. (1375-1377)

Non qu'elle fasse ici un éloge du « sadisme »; mais celui-ci, selon elle, est à distinguer radicalement d'une « initiation à certains aspects du jeu sexuel », qui n'est pas « néfaste »: « Je m'endormis contente d'avoir été trouvée belle, émue que ces minces protubérances sur ma poitrine s'appelassent déjà des seins, satisfaite aussi d'en savoir un peu plus sur ce qu'est un homme » (Yourcenar 1375-1377). La sexualité apparaît de même chez Duras comme un élément majeur du développement enfantin: sexualité incestueuse (et interdite par le « corps » familial, celui du grand frère comme celui de la mère) avec le " petit frère ", sexualité accomplie, dès la puberté, avec l'amant « chinois ». On peut enfin se demander dans quelle mesure Catherine Cusset ne se situe pas dans une perspective assez proche de ces deux auteures dans En tout innocence, qui traite 
cavalièrement du « traumatisme » qu'aurait subi Marie, puisque celui-ci n'est vécu comme tel que parce que l'institution, représentée par les instances parentale, médicale et juridique, le lui suggère.

Un premier paradoxe propre à certains romans de femmes contemporains pourrait dès lors s'énoncer ainsi: on reconnaît aux fillettes une sexualité précoce (masturbation, expériences avec d'autres fillettes comme chez Cusset [1997], autonomie des petites sorcières de Marie Ndiaye, etc.) jusqu'alors déniée, mais dans le même temps, on borde cette sexualité de tabous: le corps de l'enfant est proprement intouchable par l'adulte, c'est-à-dire sacré. Le toucher, voire le regarder avec un regard de désir, c'est tuer l'enfant, la faire passer au statut de femme: « c'est le viol de mon visage, de mes yeux, de ma peau [...]. Il finit l'enfance » (Bouraoui 43). Même chez Catherine Cusset, la masturbation apparaît d'ailleurs comme un défi à l'ordre familial. Le second paradoxe relève d'une contradiction interne au personnage de la petite fille: celle-ci, on vient de le voir, ne serait intéressante que dans la mesure où elle est une victime (moyen, on l'a dit, de donner un peu de sel à des récits souvent dépourvus de moteur narratif extérieur au vécu des protagonistes). En parallèle à la volonté affichée de rendre à la petite fille ce qui lui appartient (un corps, une indépendance, une absence légitime d'《 innocence », des fantasmes), un courant plus souterrain se laisse percevoir, qui fait de la fillette un être diminué, amoindri, et qui la rapproche de ce cliché contre lequel l'anthropologie (de Bourdieu comme d'Héritier) s'insurge précisément: la faiblesse ${ }^{\mathrm{ix}}$.

Néanmoins, ces petites filles socialement « ratées », puisqu'elles ne répondent pas aux exigences des petites filles modèles, gagnent ainsi leur droit à l'existence. En effet, autre point commun à la plupart de ces romans, la résolution des identités éclatées de ces paradoxales petites filles, réside dans l'art, territoire pourtant éminemment et séculairement masculin. Nancy Huston, dans Journal de la création, a rappelé combien 1'exclusion des femmes de l'art manifeste de domination masculine intériorisée et de dualisme: depuis Aristote, aux hommes les hauteurs de l'esprit, aux femmes l'avilissement des corps. Aux hommes la création d'une oeuvre et la postérité intellectuelle, aux femmes la parturition et la descendance matérielle. Aux hommes les enfants-textes, aux femmes les enfantschairs, analogie explicite dans l'emploi récurrent de la métaphore de la gestation et de l'accouchement appliquée aux œuvres spirituelles (que 1'on songe, dès l'Antiquité, à la maïeutique socratique, quand littéralement, maieutiké signifie accouchement). Et il va sans dire que les affres de la création spirituelle sont hautement valorisées par rapport aux souillures et déjections des accouchements naturels, selon la séculaire hiérarchie entre culture et nature, homme et femme.

Les femmes artistes, prises entre deux feux, doivent alors choisir leur camp. Cet écartèlement écartelé entre féminin (de par leur identité sexuée), et masculin (de par leur identité d'artiste) se solde par une auto-mutilation dont Simone de Beauvoir, Simone Weil ou Virginia Woolf représentent le paradigme. Si elles veulent écrire, il leur faut sacrifier et mortifier leur corps, renier l'incarnation de leur féminité, en en gommant tous les signes extérieurs ou les manifestations biologiques: c'est notamment le rôle de l'anorexie ou du refus de 
la maternité - ce sont alors leurs œuvres, comme pour leurs homologues masculins, qui leur tiennent lieu d'enfants.

Les petites filles "ratées" de nos romans réactualisent ce même dualisme du corps et de l'âme, sous-tendu par la « valence différencielle », pour reprendre le terme de Françoise Héritier, des genres. Par l'art, elles parviennent, enfin, à rejoindre le monde des hommes. Paul, anciennement Denise, trouve dans la musique, par la métaphore éjaculatoire explicite qui clôt le roman, la jouissance qui lui est refusée sexuellement. Pour Nina, pour Denise Lesur, pour Marie, ce sera l'écriture qui les sauvera de leur trouble intérieur, qui résout, enfin, leur hésitation entre deux mondes. L'art leur permet de rejoindre ces mondes interdits - sexuels, sociaux, nationaux - à la frontière desquels elles se tenaient, de passer d'un mode de refus vindicatif à un mode de création revendicatif. Mais ce qui pourrait passer pour un cliché élimé, usé jusqu'à la trame du texte, est en réalité réinterprété. En effet, ces petites filles devenues femmes n'ont plus forcément à choisir entre art et enfants ${ }^{\mathrm{x}}$ : sans entrer dans les dimensions autobiographiques des œuvres, les narratrices de Lorette Nobécourt, de Camille Laurens, d'Annie Ernaux sont des mères, heureuses de l'être. Si l'art est toujours accouchement, ce n'est plus d'enfants qu'il s'agit désormais. L'art, par la recomposition de leur identité, leur permet enfin de se faire « un nom »: c'est elles-mêmes qu'elles mettent au monde, devenant leur propre œuvre. L'écriture, la musique ne remplacent donc plus forcément les enfants, mais permettent de se créer soi-même, d'enfin trouver sa place, là où ont échoué les normes et les lois sociales. Une nouvelle variation du dualisme philosophique entre corps et âme se déploie ainsi: c'est grâce à l'art, à l'activité spirituelle, bref à l'âme, que ces petites filles « se » naissent et se reconnaissent artistes, revendiquent leur aspect " monstrueux " de femme-homme, et résolvent enfin les défaillances de leurs corps. On assiste finalement à une sorte de transubstantation, de métamorphose finale du corps en livre. Par cet acte maïeutique, elle parvient à dépasser son organisme biologique. Se faire un nom, c'est se détacher de l'inné, et, contre le patronyme hérité du père, quasiment se refaire un corps. À écrire ainsi sur leur corps, à décrire les règles de l'incorporation sociale subie lors de leur enfance, elles donnent à lire, au sens le plus fort du terme, un texte-corps. Le roman de Camille Laurens se clôt sur une analogie entre l'aspect matériel d'un livre ouvert et 1'enlacement des bras: "C'est vous, c'est bien vous sur la rive opposée, la distance entre nous se réduit, bientôt s'annule, dansons, veux-tu, je te rejoins et tu m'étreins - ah serrez-moi, emportez-moi — qu'on est bien, oui, qu'on est bien, dans ces bras-là ! » (310). Quant à Annie Ernaux, elle est extrêmement explicite: " prenez, et lisez car ceci est mon corps et mon sang qui sera versé pour vous » $(1997,41)$. 


\section{Conclusion}

La petite fille modèle du siècle précédent, corsetée dans ses robes comme dans les normes de son temps, se devait d'apprendre très tôt son métier de mère et d'épouse. En même temps qu'on lui redressait le corps, à coups de férule ou de règles de maintien, son âme et son esprit se devaient d'être ployés, tant s'imposait l'évidence de l'équivalence entre « beau » sexe et sexe « faible ». La petite fille, femme en miniature, puisque la femme restait éternellement une enfant, vivait, corporellement, mentalement, légalement, sous tutelle: tuteur des baleines de corsets (n'est-ce pas ainsi qu'on fait pousser « les belles plantes »?), tuteur légal en la personne du père, du frère, du mari, tuteur de l'âme avec le confesseur (Perrot). Les anthropologues, les sociologues, les historiens de la pensée l'ont montré: quel que soit le lieu et l'époque, les normes s'incorporent, façonnent les silhouettes, les morphologies, les attitudes, et ce sont jusqu'aux fonctions naturelles et aux sensations qui se trouvent ainsi déterminées (Détrez). Le constat a souvent été fait que la littérature française féminine contemporaine se focalise sur la description du corps dans tous ses états, repoussant à chaque rentrée littéraire les frontières du dicible. Prenant à leur compte un nouveau " sens commun savant », vulgate sociologique et psychanalytique, les auteures parviennent ainsi à montrer comment le corps des fillettes sert d'interface entre l'individu et le groupe, l'intériorité et l'extériorité. Petites filles en marge, petites filles « ratées » à l'aune de la reproduction sociale, ces enfants n'en sont que la manifestation plus évidente de la violence - qui pour être symbolique n'en est pas moins réelle (que l'on songe au dressage des corps par les régimes totalitaires) - qui, dès l'enfance, s'exerce sur les corps. Si ces petites filles paient leur résistance par une souffrance morale ou physique (aménorrhée, psoriasis,...), elles ont cependant la chance, grâce à l'art, de réconcilier leurs identités écartelées. Réinvestissant le cliché éculé de l'art comme accouchement, souvent mis au service de l'affirmation de la suprématie du cerveau masculin sur l'utérus féminin, ces auteures parviennent à le réinterpréter: l'écriture permet à ces petites filles de se faire un nom, de se faire un corps. La petite fille mal modelée devient ainsi une femme libre et indépendante, même si cet affranchissement réactive certains paradoxes classiques du dualisme entre corps et âme.

\section{Notes}

${ }^{i}$ Cet article prend place dans un travail de plus grande ampleur que nous menons conjointement, et qui vise à étudier, en tant que littéraire et sociologue, l'écriture du corps féminin dans les romans contemporains.

ii Sur ces points, voir Héritier, 2003, Bourdieu et Blöss.

iii L'hérö̈ne de Noëlle Châtelet s'appelle Denise, et choisit ensuite le prénom Paul. Pour la distinguer d'une autre Denise, celle d'Annie Ernaux, nous avons choisi par commodité d'accoler les deux prénoms.

iv La différence centrale de ce roman avec les œuvres que nous envisageons dans cet article étant que chez Ernaux, c'est le personnage de la mère qui la pousse à 
récuser les traditions, par un désir de surclassement social dont sa fille souffrira plus tard mais qui lui permettra de rendre hommage à celle qui lui a permis d'échapper au destin tout tracé de la femme du peuple (mariage, enfants, foyer). ' Que l'on pense aux analyses menées par Michel Foucault, sur le rôle de ces institutions que sont l'école, l'armée et l'hôpital comme instances de diffusion des normes et de la discipline.

vi Françoise Héritier explique la valence différentielle des sexes par la capacité de la femme à porter du différent (à savoir du masculin) et par l'incapacité des hommes à porter des enfants (et donc à autoreproduire son genre).

vii Voir notamment l'incipit de La Sorcière. Que leur seul acte véritable (à part la transformation en corneilles) soit d'aider leur tante à avorter est évidemment symptomatique d'un refus de conformer le passage à l'âge adulte à la trop classique capacité de gestation.

viii Il y a évidemment des exceptions à cette remarque, notamment chez Pierrette Fleutiaux, qui récuse cette culpabilisation acharnée de la mère moderne: la femme de l'ogre refait sa vie... avec Poucet. Quant au Petit Pantalon Rouge, élevé par une mère et une grand-mère libérées, il s'appuiera sur leurs conseils et leur comportement pour s'assurer le pouvoir sur les loups.

ix Elisabeth Badinter montre bien la tentation récurrente dans les discours d'une « victimisation » de la femme.

x Encore faudrait-il, mais ce serait dépasser les bornes de la " petite fille », analyser comment le refus relatif de la maternité s'est déplacé en rapport ambigu: homosexualité chez Nina Bouraoui ou Amélie Nothomb, sexualité débridée chez Catherine Cusset, Lorette Nobécourt, Annie Ernaux, Camille Laurens, etc.

\section{Bibliographie}

Badinter, Elisabeth. Fausse route. Paris: Odile Jacob, 2003.

Blöss, Thierry (dir.). La Dialectique des rapports hommes-femmes. Paris: PUF, 2001.

Bouraoui, Nina. Garçon manqué. Paris: Stock, 2000.

Bourdieu, Pierre. Le Sens pratique. Paris: Minuit, 1980.

__ La Domination masculine. Paris: Seuil, 1998.

Châtelet, Noëlle. La Tête en bas. Paris: Seuil, 2002.

Cusset, Catherine. En toute innocence. Paris: Gallimard, 1995.

- Jouir. Paris: Gallimard, 1997.

Détrez, Christine. La Construction sociale du corps. Paris: Seuil, 2002.

Didier, Béatrice. L'Ecriture-Femme. Paris: PUF, 1981.

Ernaux, Annie. Les armoires vides. Paris: Gallimard, 1974.

___ Ce qu'ils disent ou rien. Paris:Gallimard, 1977.

La Femme gelée. Paris: Gallimard, 1981.

— La Honte. Paris: Gallimard, 1997.

Fleutiaux, Pierrette. Métamorphoses de la reine. Paris: Gallimard, 1984.

Foucault, Michel. Surveiller et punir. Paris: Gallimard, 1975. 
Goffman, Erving. "La ritualisation de la féminité ». Actes de la recherche en sciences sociales, $\mathrm{n}^{\circ} 14,1977$.

Heinich, Nathalie. États de fernme. Paris: NRF Essais, Gallimard, 1996.

Héritier, Françoise. Masculin/Féminin, t. I et II. Paris: Odile Jacob, 1996, 2003. Huston, Nancy. Journal de la création. Paris: Babel, 1990.

Kaufmann, Jean-Claude. Corps de femmes, regards d'hommes. Paris: Nathan, 1995.

Laurens, Camille. Dans ces bras-là. Paris: P.O.L., 2000.

Ndiaye, Marie. La Sorcière. Paris: Minuit, 1996.

Nobécourt, Lorette. Nous. Paris: Pauvert, 2002.

Nothomb, Amélie. Métaphysique des tubes. Paris: Albin Michel, 2002.

Pagès, Michèle. "Corporéités sexuées, jeux et enjeux ». In La Dialectique des rapports hommes-femmes. Thierry Blöss (dir.). Paris: PUF, 2001. 219-234.

Perrot, Philippe. Le Travail des apparences. Le corps féminin XVIIIe-XIXe siècle. Paris: Seuil, 1984.

Yourcenar, Marguerite. Quoi ? L'éternité. Paris: Gallimard, 1982. 1375-77. 